\title{
Pricing Model and Compensation Strategy of Wind Power AGC Ancil- lary Services Based on the Day-ahead Market and Priority Decision- making Model
}

\author{
Zhang Yuzhuo ${ }^{1 *}$, Wang Ling ${ }^{2}$, Zhao Xin-gang ${ }^{1}$ \\ ${ }^{1}$ School of Economics and Management, North China Electric Power Univ., Beijing 102206, China \\ ${ }^{2}$ State Grid Liaoning Electric power co., LTD. Benxi Power Supply Company, Benxi, 117000, China
}

846689602@qq.com

Keyword: Wind power; Ancillary services; AGC; Compensation strategy

\begin{abstract}
That wind power provides automatic generation control (AGC) is an innovative solution to the lack of AGC ancillary services and the problem of abandoned wind. How to appropriately compensate the wind power generators who provide AGC ancillary services is a critical issue. Based on the day-ahead market and priority decision-making model, this paper takes two sciences, unit performance preference and unit economic preference, into consideration, and uses the comprehensive evaluation method to order wind power farms. Then it establishes pricing model and compensation strategy according to the capacity price and the electricity price. Aim to provide a reference for future pricing and compensation model of wind farms in Chi-na.
\end{abstract}

\section{INSTRUCTION}

Automatic generation control (AGC) is a key part of auxiliary services, which is of great importance to maintain the security and stability of power grid [Zeng Ming, 2011]. Only the power suppliers possess AGC units in the electricity market environment, therefore, it is necessary to build an appropriate AGC ancillary services pricing model and compensation mechanism to stimulate the wind power farms to provide safe and economical AGC ancillary service.

The pricing and compensation of AGC mainly include cost pricing and market pricing. Traditional AGC cost pricing generally takes the investment cost, maintenance cost, operation cost and opportunity cost of a unit into consideration [Wang Juanjuan, 2010] [Tao Chunhua, 2006]. Due to the incomplete information, the cost of AGC units to provide ancillary services is difficult to measure. However, cost pricing method provides a theoretical basis for the market pricing. Using probabilistic method and the equivalent price principle to stochastic simulation of AGC units is able to estimate the value of the capacity of ancillary services and the loss of opportunity cost that AGC units could provide, and these two values can be understood as capacity of AGC units in the market for electricity and power electricity [Yan Maosong, 2012].

The standard value of AGC that the power plant adjusts with the obligation could be made according to the situation of power load characteristics and AGC adjustment, and the grid AGC deal making model can be established [Xia Wei, 2012]. It is one of the typical methods of market pricing. Li Dan (2010) constructed comprehensive index evaluation of AGC performance, and proposes the corresponding AGC compensation method. ORQUINRA, (2004) established the corresponding indicators including conditions (such as market supply and demand ratio), behavior (such as the retention ratio) and performance (such as market efficiency index in view of the ancillary services market competition, and make a comprehensive evaluation about the auxiliary services of bidding unit (AGC and spinning reserve, etc.). Comprehensive evaluation method was applied to AGC ancillary service market in the United States, ISO determines the market clearing price, AGC service FM on the basis of AGC capacity quotation the tenderer provide, according to the order of the final price [Zhang Ji, 2006]. MU Tao (2005) and Wu Jigguang (2006) constructed index model with analytic hierarchy process, sort the bidding of AGC units, and finally compare the result of the different scheme of compensation. 
China's power auxiliary service market has not been established and the wind turbines have the ability to provide security and stability of AGC service are relatively scarce, AGC priority decision model has more practical significance, at the same time in order to make the AGC trading not too complex, this paper chose the day-ahead market bidding model. Using the analytic hierarchy process to evaluate the unit only is too subjective, and entropy weight method is to evaluate objective data. The comprehensive evaluation that combines both of them are more persuasive. In addition, wind unit capacity is small, in commonly $2 \mathrm{MW}$ or so, thus based on the wind farm, this paper evaluates wind farm with the final integration method.

\section{THE DESCRIPTION OF THE PROBLEM}

In the ancillary services market, part of the wind turbines has the ability to provide AGC ancillary service. Wind AGC units in wind farms bid in the day-ahead market, and report only once each work day. In the model of priority in electric power market decisions, the winning wind farms can't participate in the spot electricity market auction, only wind farms out of AGC bidding system could participate in.

Analogy to the traditional ancillary services markets, wind farms for the bidding process as showed in Figure 1[Li Yanqing, 2012] [Huang Yonghao, 2003]: (1) Quotation. Wind farms participated in AGC service declare the original data within daily prescribed period of time. Declare content includes electricity price, electricity price, availability, capacity adjustment capacity, adjust the rate, adjusting precision and response time. For the convenience of calculation, bid price unit is the yuan/MWh. (2) Initial screening. If the wind farm's availability is lower than a certain value (e.g., 98\%) , dispatch center kick it out of the bidding according to the power grid hard Numbers. (3) Wind farm sorting. The remaining of wind farms are sorted based on the system of comprehensive evaluation results. (4) Choose a wind farm. Combining with the AGC day-ahead capacity demand that prediction center has forecast (the day-ahead demand forecast should be more than 15 minutes), select wind farm from high to low according to the wind farm sort. Wind farms which were not selected also withdraw from the bidding. (5) Transfer. Transfer wind farms who won the bid according to AGC 15 minutes' capacity demand forecasting. Capacity that was not transferred cannot participate in electricity market auction. (6) Compensation and settlement. Compensate winning wind farms based on their own capacity of the respective quotation and adjustable capacity. Compensate for capacity electricity according to the actual transfer of wind farms' capacity.

Shown as the Fig. 1, after all wind farms meet the requirements participating in the bid, how do we determine the corresponding sorted basis to choose wind turbines to provide AGC service is the primary problem. After the unit is transferred how to compensate for has important effect on the safety and economy of power grid. So below will build pricing model and compensation strategy of wind turbines providing AGC service based on the two key points. 


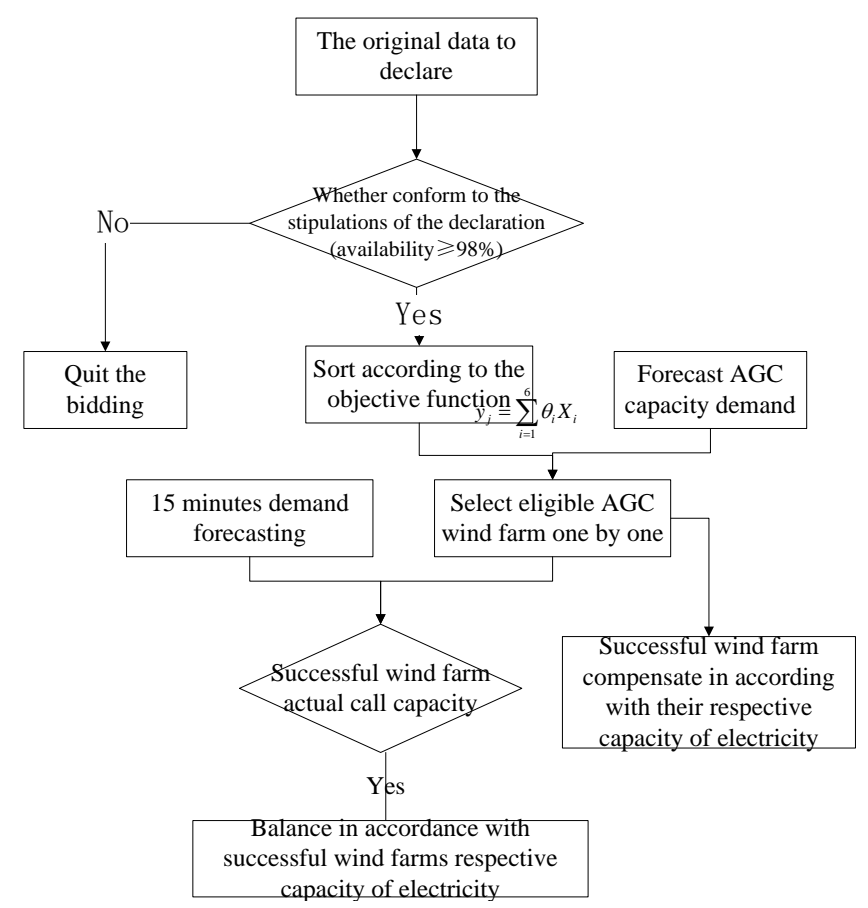

Figure 1. The flow chart of AGC auction.

\section{PRICING MODEL}

In this paper, wind farm bidding methods mainly are analytic hierarchy process, eigenvalue method, entropy weight method and integration method [He Yongxiu, 2011].

\subsection{The standardization of data processing}

Indicators are with dimension, and the numerical difference between the absolute value is large. To eliminate its influence on the selection of AGC wind farms, before calculating objective weight with entropy weight method, various indicators need the dimensionless normalized processing. Suppose there are m AGC wind bids, by the North China grid power plant auxiliary service and parallel operation management rules through by north China region and the related literature, number of evaluation index of AGC wind farm is a total of six, which form the original data matrix: $X=\left(X_{i j}\right)_{m \times 6}$.

Among them, greater the value of the adjusting speed $\left({ }^{X_{3}}\right)$, and adjustable capacity $\left({ }^{X_{3}}\right)$, greater the results of comprehensive evaluation results; Higher the index value of capacity of electricity $\left({ }^{X_{1}}\right)$, electricity price $\left({ }^{X_{2}}\right)$, adjusting precision $\left({ }^{X_{4}}\right)$ and response time $\left({ }^{X_{5}}\right)$, more bad the result of comprehensive evaluation. So can make:

Get normalized matrix:

$$
\begin{aligned}
& R_{i j}=\frac{X_{i j}-\min _{i} X_{i j}}{\max _{i} X_{i j}-\min _{i} X_{i j}}, j=3,6 \\
& R_{i j}=\frac{\max _{i} X_{i j}-X_{i j}}{\max _{i} X_{i j}-\min _{i} X_{i j}}, j=1,2,4,5
\end{aligned}
$$

$$
X^{\prime}=\left(X_{i j}\right)_{m \times 6}
$$

\subsection{The entropy weight method to determine the objective weight}

Entropy weight method is a kind of objective methods of empowerment. In information theory, entropy is the measure of system disorder and source signal uncertainty; If the index information entropy is smaller, the amount of information indicators provide, the effect in the comprehensive evaluation and its weight or entropy is greater. 
According to the matrix $X^{\text {' }}$, calculate the scheme $i$ 's contribution $P_{i j}$, under the index $j$ 's attribute, among them $P_{i j}=X^{\prime}{ }_{i j} / \sum_{i=1}^{m} X^{\prime}{ }_{i j}$. Calculate the entropy value $e_{j}$ of index $j, e_{j}=-k \sum_{i=1}^{6} P_{i j} I n P_{i j}$ (when $P_{i j}=0$, make $\lim _{P_{i j} \rightarrow \infty} P_{i j} \operatorname{In} P_{i j}=0$ ), among them, $k=1 / m$. Calculate the Coefficient of variance of the index $j, g_{j}=1-e_{j}$ and determine the normalized weight is $r_{j}=g_{j} / \sum_{j=1}^{m} g_{j}$

\subsection{The characteristic value method to determine the subjective weight}

In this paper, we use capacity of electricity, electricity tariff, speed, accuracy, response time, and adjustable capacity of six factors as evaluation index of AGC wind farm, with $X_{1}, X_{2}, X_{3}, X_{4}, X_{5}$ and ${ }^{X_{6}}$ to respectively represent. Hierarchical analysis model is set up as follows:

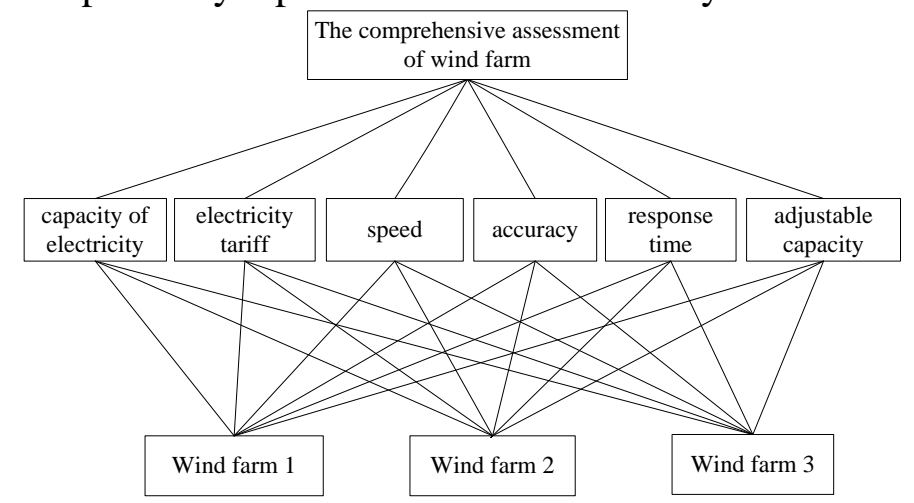

Figure 2. Hierarchy model of assessing AGC wind farms.

When compare the element ${ }^{i}$ and element ${ }^{j}$ with the importance of some factors on the upper layer relatively, use the quantification relative weight $a_{i j}$ to describe. In this paper, there are total of six elements in the comparison, so $A=\left(a_{i j}\right)_{6 \times 6}$ is called a paired comparison matrix. In paired comparison matrix ,value of $a_{i j}$ refer to Satty 's proposal, value between 1 to 9 and its inverse.

Steps of inspecting consistency of paired comparison matrix A in general literatures are as follows: firstly, measure a paired comparison matrix A's inconsistent degree index CI:

$C I=\left(\lambda_{\max }(A)-6\right) /(6-1)$.

Then get standard value of $R I$ by looking up table, and calculate the random consistency ratio CR of comparison matrix A, $C R=C I / R I$. When $C R<0.1$, judge that comparison matrix A has satisfactory consistency, or its inconsistent degree is acceptable; When does not meet $C R<0.1$, judge that comparison matrix A has no satisfactory consistency, need to be revised until it has satisfactory consistency, and get all of the same level indicators' sorting weight value ${ }^{w_{j}}$ for the highest levels of importance.

\subsection{Based on integration method of the combination of empowerment}

Combine the objective weight $r_{j}$ with subjective weight ${ }^{w_{j}}$, and the combination of empowerment get is :

$$
\theta_{j}=r_{j} \cdot w_{j} / \sum_{j=1}^{6} r_{j} \cdot w_{j}
$$

so the comprehensive evaluation index $i$ for AGC wind farm is

$$
y_{i}=\sum_{j=1}^{6} \theta_{j} X_{j}^{\prime}
$$




\section{THE COMPENSATION STRATEGY}

Evaluate AGC unit contribution comprehensively, and provide AGC ancillary service with compensation basis respectively. The method is based on daily 96 points every day (15 min each time), according to the capacity cost and power cost to compensate.

Review units according to performance, quotation and the actual power indicator submitted by AGC wind farm, then the inspection qualified units shall be compensated. The $i$ th bid AGC wind farm should pay ${ }^{C_{i}}$ :

$$
C_{i}=P_{c i} Q_{i} / 4+K_{i} P_{e i} \int_{0}^{1 / 4} R_{i} d t
$$

Simplify the above as follows:

$$
\begin{aligned}
& \left(P_{c i} Q_{i}+K_{i} P_{e i} Q_{i}^{*}\right) / 4 \\
& i=1,2,3 \cdots \cdots m-1: Q_{i}^{*}=Q_{i} ; \\
& \left.i=m: Q_{i}^{*}=Q_{15 \min }-\sum_{i=1}^{m-1} Q_{i}^{*}\right) \\
& Q_{15 \min }=\sum_{i=1}^{m} Q_{i}^{*} \leq Q_{d} \leq \sum_{i=1}^{m} Q_{i}
\end{aligned}
$$

$T$ is Operation of AGC time, $R_{i}$ is regulating capacity recorded in EMS. $Q_{i}$ is the adjustable capacity, $Q_{i}^{\cdot}$ is each wind farm's actual call capacity in 15 minutes, $P_{c i}$ is the $i$ th wind farm's capacity of electricity, $P_{e i}$ is the $i$ th wind farm's electricity tariff, $K_{i}$ is the $i_{\text {th }}$ wind farm's AGC availability. $Q_{d}$ is day-ahead capacity demand forecast of AGC, $Q_{15 \min }$ is capacity demand forecast of AGC in 15min.

\section{CONCLUSION}

According to different scheduling preference, this paper discussed the AGC service on wind farm, and put forward wind farm bidding and compensation strategy in day-ahead market priority decision mode. Combine the entropy weight method of objective weight combination weights, when play down the difference of the subjective preferences, also strengthen the characteristics of the raw data itself, therefore, it makes results more reliable, more persuasive.

\section{ACKNOWLEDGMENT}

This article is supported by "Science and technology project funding by State Grid Liaoning electric power co., LTD. Benxi Power Supply Company” (Grant No. FZJS1400820 and FZJS1400825) and "Science and technology project funding by State Grid Corporation".

\section{REFERENCES}

[1] Zeng Ming. 2011. Power demand side response theory and its application in electric power market. Beijing: China Electric Power Press.

[2] Wang Juanjuan et al. 2010. The cost of AGC ancillary service in power market research was reviewed. Power system protection and control 38(24): 56-61.

[3] Tao Chunhua et al. 2006. AGC cost research electric power market environment. East China Electric Power 4(34):5-6.

[4] Yan Maosong et al. 2003. Probability, a new method of AGC prior pricing and a posteriori assessment. Automation of electric power systems 27(2):1-6. 
[5] Xia Wei. 2012. Large-scale wind power grid AGC trade research. Water and electricity energy science 30(04): 195-198.

[6] Li Dan et al. 2010. Interconnection of power plant management appraisal system, AGC regulating performance compensation measures. Automation of electric power systems 34(4):107-111

[7] ORQUINRA et al. 2004. An option evaluation model for ancillary and balancing services. Proceedings of IEEE PES Power Systems Conference and Exposition, New York, USA: 78-83.

[8] Zhang Ji et al. 2006. Based on the SCP and fuzzy algorithm of market power threat level assessment. Automation of electric power systems 30(16):15-20.

[9] MU Tao et al. 2005. Competitiveness criterion and evaluationapproach of AGC market. Proceedings of IEEE/PES Transmission and Distribution Conference \& Exhibition, Dalian, China: 1-5.

[10] Wu Jigguang ea al. 2006. The AGC bidding auction algorithm based on AHP. Inner Mongolia electric power technology 24(03):5-8.

[11] Li Yanqing et al. 2012. AGC units compensation method based on fuzzy analytic hierarchy process (ahp) is studied. Electric power science and engineering 28(11):22-27.

[12] Huang Yonghao. 2003. Power auxiliary service market operation mechanism and model. Automation of electric power systems 27(2):33-36

[13] He Yongxiu. 2011. Comprehensive evaluation method and application of electric power. Beijing: China Electric Power Press. 